University of Wollongong

Research Online

Faculty of Social Sciences - Papers (Archive) Faculty of Arts, Social Sciences \& Humanities

$1-1-2020$

Social anhedonia and social functioning: Loneliness as a mediator

Melody Tan

University of Wollongong, acmt058@uowmail.edu.au

Amy Shallis

University of Wollongong

Emma Barkus

University of Wollongong, ebarkus@uow.edu.au

Follow this and additional works at: https://ro.uow.edu.au/sspapers

Part of the Education Commons, and the Social and Behavioral Sciences Commons

Research Online is the open access institutional repository for the University of Wollongong. For further information contact the UOW Library: research-pubs@uow.edu.au 


\title{
Social anhedonia and social functioning: Loneliness as a mediator
}

\begin{abstract}
2020 The Institute of Psychology, Chinese Academy of Sciences and John Wiley \& Sons Australia, Ltd Social anhedonia is associated with reduced social functioning and diminished reward from social interactions. Individuals expressing social anhedonia are likely to experience reduced social connectedness and feel lonely. Loneliness is also associated with reduced social functioning. Therefore, loneliness could account for the relationship between social anhedonia and social functioning. We aimed to determine whether loneliness mediates the relationship between social anhedonia and reduced social functioning. In total, 824 young adults ( $\mathrm{M}$ age $=21.03, \mathrm{SD}=5.59 ; 72.3 \%$ female) completed the RevisedSocial Anhedonia Scale (RSAS), University of California Los Angeles (UCLA) Loneliness Scale, and the Social Functioning Scale (SFS). Scores on thee SFS were summed into six subscales: Social Withdrawal, Relationships, Social Activities, Recreational Activities, Independence (Competence), and Independence (Performance). Negative affect (Depression, Anxiety and Stress Scales-21 [DASS] total score) was included as a covariate. Both the RSAS and the UCLA Loneliness Scale were negatively correlated with overall and all social functioning subscales. The DASS-21 positively correlated with all variables of interest. Mediation analyses revealed that loneliness partially mediated the relationship between social anhedonia and the social functioning subscales, with the exception of Recreational Activities. However, loneliness was a full mediator for the relationship between social anhedonia and overall social functioning. The study findings suggest that targeting loneliness in interventions may be important for improving various aspects of social functioning in those individuals who express social anhedonia.
\end{abstract}

\section{Disciplines}

Education | Social and Behavioral Sciences

\section{Publication Details}

Tan, M., Shallis, A. \& Barkus, E. (2020). Social anhedonia and social functioning: Loneliness as a mediator. PsyCh Journal, 
Running head: LONELINESS, SOCIAL ANHEDONIA AND SOCIAL FUNCTIONING 1

Social anhedonia and social functioning: Loneliness as a mediator

Melody Tan, Amy Shallis, Emma Barkus

University of Wollongong 


\begin{abstract}
Social anhedonia is associated with reduced social functioning and diminished reward from social interactions. Individuals expressing social anhedonia are likely to experience reduced social connectedness and feel lonely. Loneliness is also associated with reduced social functioning. Therefore, loneliness could account for the relationship between social anhedonia and social functioning. We aimed to determine whether loneliness mediates the relationship between social anhedonia and reduced social functioning. In total, 824 young adults (mean age $=21.03(\mathrm{SD}=5.59) ; 72.3 \%$ female) completed the Revised-Social Anhedonia Scale (RSAS), University of California Los Angeles (UCLA) Loneliness Scale and the Social Functioning Scale (SFS). Scores on SFS were summed into six subscales: Social withdrawal, Relationships, Social activities, Recreational activities, Independence (Competence) and Independence (Performance). Negative affect (Depression, Anxiety and Stress Scales-21 total score) was included as a covariate. Both RSAS and UCLA were negatively correlated with overall and all social functioning subscales. DASS positively correlated with all variables of interest. Mediation analyses revealed that loneliness partially mediated the relationship between social anhedonia and the social functioning subscales, with the exception of recreational activities. However, loneliness was a full mediator for the relationship between social anhedonia and overall social functioning. The study findings suggest targeting loneliness in interventions may be important for improving various aspects of social functioning in those individuals who express social anhedonia.
\end{abstract}

Keywords: Loneliness; Social Anhedonia; Social Functioning

Social anhedonia and social functioning: Loneliness as a mediator 
Social anhedonia (SA), a trait-like disinterest in interpersonal interactions, is often characterised by reduced ability to experience pleasure in social situations (Brown, Silvia, Myin-Gerneys, \& Kwapil, 2007; Horan, Green, Kring, \& Nuechterlein, 2006). SA is related to but distinct from introversion (Martin, Cicero, Bailey, Karcher, \& Kerns, 2015; Ross, Lutz, \& Bailey, 2002) and social anxiety (Brown et al., 2007; Brown, Silvia, Myin-Gerneys, Lewandowski, \& Kwapil, 2008). Those who express SA withdraw from social interactions due to a lack of reward from these experiences rather than heightened levels of anxiety induced by social circumstances. SA is present in both non-clinical and clinical populations. The expression of SA in psychiatric disorders (Fortunati et al., 2015; Blanchard, Horan, \& Brown, 2001) and the general population (Healey, Morgan, Musselman, Olino, \& Forbes, 2014; Setterfield, Walsh, Frey, \& McCabe, 2016) has relevance for social outcomes, including levels of social functioning. Given the key role for social support in maintaining well-being, it is imperative that factors which impinge upon social functioning are fully understood.

SA has particularly been considered as a characteristic of the psychosis continuum (Kwapil, 1998). Increased SA is a robust vulnerability marker for future development of psychotic disorders (Kwapil, 1998; Velthorst et al., 2009). Further, elevated ratings of SA have repeatedly been reported by individuals with schizophrenia as well as amongst relatives of patients with schizophrenia, when compared to the general population without genetic liability (Blanchard, Gangestad, Brown, \& Horan, 2000; Gooding, Davidson, Putnam, \& Tallant, 2002; Schurhoff et al., 2003). Within the general population and patients with schizophrenia, expression of SA is associated with symptoms of depression (Joiner, Brown, \& Metalsky, 2003; Rey, Jouvent, \& Dubal, 2009), perhaps indicating that SA is transdiagnositic and therefore has implications beyond psychotic disorders. 
Indeed, SA is reported by individuals with various disorders, including schizoaffective disorder, eating disorders, autism-spectrum disorder, posttraumatic stress disorder, and substance-use disorder (Frewen, Dozois, \& Lanius, 2012; Gooding, Tallent, \& Matts, 2005; Harrison, Mountford, \& Tchanturia, 2014; Novacek, Gooding, \& Pflum, 2016; Treadway, \& Zald, 2011). SA is not bound by diagnosis but rather may have neurocognitive and genetic underpinnings which increase vulnerability for reduced reward from social interactions, independent from any one psychological disorder (Bedwell, Gooding, Chan, \& Trachik, 2014). The presence of SA may be one factor which contributes to the maintenance of impaired social functioning in psychological disorders and predicts poor treatment response (Spijker, Bijl, de Graaf, \& Nolen, 2001). Understanding the nature of social anhedonia is becoming increasingly important since researchers and clinicians agree that successful treatment response involves recovery from both clinical symptoms and having adaptive social functioning (Brissos, Molodynski, Dias, \& Figueira, 2011; Juckel, \& Morosini, 2008).

With an emphasis on social outcomes, higher levels of SA are associated with impaired social functioning including reduced social support, reduced willingness to engage in social interactions, greater level of perceived stress and poorer interpersonal relationships especially within the family unit (Blanchard, Collins, Aghevli, Leung, \& Cohen, 2011; Horan, Brown, \& Blanchard, 2007; Llerena, Park, Couture, \& Blanchard, 2012). It is widely reported that reduced social support and poor social networks are associated with clinically poor significant health outcomes (Holt-Lunstad, Smith, \& Layton, 2010; House, 2001; Miller, Chen, \& Cole, 2009). Despite their self-reported preferences to be alone, individuals with SA report less satisfaction with the social support they receive (Brown et al., 2007; Blanchard et al., 2011). Collectively, this suggests a disconnection between their self-reported preferences for aloneness and innate social needs. High levels of SA are associated with high levels of 
loneliness (Badcock, Barkus, Cohen, Bucks, \& Badcock, 2016), suggesting that although social interactions are neither pursued nor pleasurable, an absence of meaningful social connections does increase the risk of loneliness for these individuals.

Loneliness is a complex negative emotional response to perceived social separateness, when there is subjective discrepancy between the desired and perceived quality of one's social relationships (Hawkley, \& Cacioppo, 2010; Peplau, \& Perlman, 1982). Loneliness has gained recent research attention as a transdiagnostic factor that increases symptom severity and maintains diagnostic status (Lim, \& Gleeson, 2014). Loneliness is related to depression (Jaremka et al., 2014), social anxiety disorder (Lim, Rodebaugh, Zyphur, \& Gleeson, 2016), and obsessive-compulsive disorder (Timpano, Cek, Rubenstein, Murphy, \& Schmidt, 2014). Interestingly, loneliness is only weakly correlated with size of social network and frequency of interactions with others (Cornwell, \& Waite, 2009); one can feel lonely despite being in a crowd or having relatively rich social lives. The key factor, therefore, appears to be the subjective perceptions of social support rather than the number or real-world objective nature of the support available to an individual (Uchino, Bowen, Carlisle, \& Birmingham, 2012). Humans are social beings by nature and have a fundamental need to belong (Baumeister, \& Leary, 1995; Lavigne, Vallerand, \& Crevier-Braud, 2011). Consequently, feelings of loneliness increase risk for detrimental psychological and physical health (Danese et al., 2009; Heinrich, \& Gullone, 2006). Therefore, it becomes important to consider whether individuals who express SA are prone to experiencing loneliness. Given a reduced desire to engage in social situations, it is possible that individuals who express SA do not feel lonely. Conversely, the reduced sense of reward and withdrawal from social situations by those with SA may be more complex than we suppose, and as such, those with social anhedonia may report higher levels of loneliness. 
To date, much of the existing literature concerning the nature of the relationship between SA and social functioning has focused on the role of cognitive (e.g. working and episodic memory, attentional control) and social cognitive processes such as theory of mind (Dodell-Feder, Tully, Lincoln, \& Hooker, 2014; Strauss, \& Gold, 2012). An alternative possibility, that would carry important treatment implications, is that SA is related to social functioning, at least partially, through loneliness. Even though previous research has yet to explore the relationship between SA and loneliness, SA co-occurs with loneliness (Lim, \& Gleeson, 2014) and loneliness is associated with anhedonia (Badcock et al., 2015). Based on existing findings, this study seeks to investigate whether higher levels of SA and loneliness will be related to lower levels of social functioning. In addition, we will examine whether loneliness mediates the relationship between SA and different aspects of social functioning.

\section{Method}

\section{Participants}

A sample of 824 participants (596 females, 228 males) was recruited through the School of Psychology research participation scheme and posters placed around the campus. The total sample has a mean age of $21.03(\mathrm{SD}=5.592)$ years, with majority of participants $(95.6 \%)$ in the age range between 17 and 35 years. This study uses opportunity sampling with no specific inclusion or exclusion criteria.

\section{Measures}

Basic demographics questions gathered information regarding the age and sex of the participants.

Revised Social Anhedonia Scale (RSAS) (Eckblad, Chapman, Chapman, \& Mishlove, 1982): 
The RSAS is a 40 true-false items, self-report inventory which assess one's capacity to experience pleasure in social interactions. This RSAS has good discriminant validity from measures of social anxiety (Cicero, Krieg, Becker, \& Kerns, 2016). Higher scores reflect greater social anhedonia.

Social Functioning Scale (SFS) (Birchwood, Smith, Cochrane, Wetton, \& Copestake, 1990):

The SFS assesses across various key skills and performance crucial for functioning in the community. In a recent review, the SFS was considered as one of the most frequently cited self-report scales (Burns, \& Patrick, 2007; Leifker, Patterson, Heaton, \& Harvey, 2011). The SFS was constructed with the aim of measuring the various aspects of functioning essential for community-dwelling in individuals with schizophrenia, including social withdrawal, interpersonal relationships, participation in activities, independence and employment. However, the SFS measures diverse and subtle aspects of social behaviours which are independent of diagnosis and are less influenced by the direct effects of clinical symptoms (Hellvin et al., 2010), therefore it is suitable for capturing social functioning in those who expresses social anhedonia (Henry, Bailey, \& Rendell, 2008). The SFS consists of 76 items with varying response formats including dichotomous questions and Likert scales. These items are spread across seven subscales including Social Withdrawal, Interpersonal and Relationships, Social Activities, Recreational Activities, Independence-Competence, Independence-Performance and Employment. As the sample population in this study comprises undergraduates who are not working full-time, the subscale of employment/occupation was excluded from the total score.

Revised UCLA Loneliness Scale Version 3 (UCLA) (Russell, Peplau, \& Cutrona, 1980):

The UCLA is a 20-item self-report questionnaire designed to measure one's subjective feelings of loneliness as well as feelings of social isolation. It requires participants 
to indicate on a 4-point Likert scale how often each statement applies to them, with 1 indicating never to 4 indicating often.

Depression, Anxiety and Stress Scale (DASS-21) (Lovibond, \& Lovibond, 1995b):

As adapted from the original 42-item version (Lovibond, \& Lovibond, 1995a), the DASS-21 is a 21-item self-report instrument designed to measure three negative emotional states of depression, anxiety, and stress. Each subscale comprises 7 items and all items are measured on a 4-point Likert scale, ranging from 0 (did not apply to me at all) to 3 (applied to me very much). Total DASS scores rather than subscales scores will be used in the analyses as they produce a composite measure of negative emotional symptoms.

\section{Procedure}

This study received ethical approval through the Social Science and Humanities Human Research Ethics Committee. Over a two-year period, undergraduate students who signed up for this study through the psychology student participation pool were given direct access to the online survey. Emails with the survey link were sent to others who were recruited through alternative means such as snowballing. Participants provided informed consent through accessing and submitting their responses online for use in the current study. Upon completion, students who undertake psychology subjects received a total of 1 course credit point.

\section{Results}

Demographic information of the 824 participants recruited and the descriptive statistics of the measures are presented in Table 1. There were no significant effects for gender, with females and males receiving similar scores across measures including social anhedonia $(t(822)=0.10, p=.92)$, loneliness $(t(822)=-0.93, p=.35)$ and negative affective 
states $(t(471.92)=1.52, p=.13)$. However, there was a significant effect for gender for social functioning $(t(822)=-4.02, p<.001)$ with females $(M=151.45, S D=21.49)$ reporting higher scores than males $(M=144.58, S D=23.18)$.

As hypothesised, social anhedonia and loneliness are both negatively correlated with social functioning (Table 2). Individuals who have greater diminished interest in social interactions or those who perceive themselves to be more disconnected from others will not have as high levels of social functioning as those who scored low on RSAS and UCLA. Negative emotional states, as measured by DASS, moderately correlated with social anhedonia, loneliness and social functioning, which emphasized the importance of including the total DASS score as a covariate in the mediation models.

The role of loneliness in mediating the relationship between SA and social functioning is illustrated in Figure 1. The significance of the indirect effect was tested using nonparametric bootstrapping analyses (Preacher, \& Hayes, 2004; Preacher, Rucker, \& Hayes, 2007) with 10,000 bootstrap samples. Table 3 presents the results from the PROCESS models testing the mediating effect of loneliness on the relationship between SA and social functioning, as well as its various subscales. DASS total score were included as covariates in the analysis.

The unstandardized regression coefficient for path $a$ was positive and significant $(b=$ $0.38, \mathrm{SE}=0.04, t(820)=8.86, p<.001)$, indicating that higher levels of SA are associated with a greater sense of loneliness. Loneliness was found to be a significant mediator between SA and all subscales of social functioning, with the exception of Recreation Activities (Sobel's test $(Z=0.006, p=1.00))$. For the other mediation analyses, direct effects were reduced but remained significant, suggesting loneliness was only a partial mediator. The biascorrected bootstrap 95\% confidence interval did not contain zero, indicating the indirect 
impact of social anhedonia on social functioning through loneliness was statistically significant.

However, loneliness was a full mediator for the relationship between SA and overall social functioning. When loneliness was introduced, the direct effect of SA to loneliness decreased $\left(c=-.28\right.$ to $\left.c^{\prime}=-.00\right)$ and became non-significant $(t(820)=0.2, p=.99)$, with a $95 \%$ confidence interval from -0.20 to 0.21 .

\section{Discussion}

In line with previous research, the results demonstrate similar patterns with regards to the negative relationship between social anhedonia, loneliness and social functioning. Individuals who experience greater feelings of loneliness and diminished pleasure from social situations reported that they function less well in social situations. Indeed, social anhedonia has been reported to be associated with poor functioning in both schizophrenia patients and those from the community population (Blanchard et al., 2011). These results are consistent with previous research which suggests loneliness is a risk factor for poor social functioning (Llerena et al., 2012). In keeping with previous research (Lim, \& Gleeson, 2014), higher levels of social anhedonia were associated with higher levels of loneliness in our sample. The mediation analyses support that social anhedonia is related to lower social functioning, at least partially, through subjective loneliness. The results in the current study were also present while controlling for the presence of negative affect which is important given that negative affect was moderately correlated with all the variables of interest.

Loneliness mediates the relationship between social anhedonia and the SFS subscales, with the exception of Recreation Activities. This finding could be explained by considering 
the types of activities found on the Recreation Activities subscale. Other subscales involve activities which require an individual to interact with other people. However, recreation activities include items which could either be completed alone or within a situation where interactions with others are either structured or can be kept to a minimum. It also includes activities where others might be involved but people complete actions alongside one another rather than through direct interaction. Both higher levels of loneliness and social anhedonia were associated with lower scores on Recreation Activities. However, one interpretation of the mediation model result, is that loneliness does not interfere with the likelihood of people engaging in recreation activities. It means that people who express social anhedonia, could be encouraged to be involved in a personal hobby such as watching movies, reading and exercising alongside other people, without their poor sense of connection to others hindering engagement. This is important because loneliness can act as a barrier for some people seeking out social interactions. Further research is needed to investigate whether those with social anhedonia have preferences for certain types of social environment in order to determine what activities could be suggested and encouraged during psychological interventions.

Given that higher levels of social anhedonia were associated with loneliness, it is possible that changing perceptions of poor connectedness with others could improve the experience of social situations for those with social anhedonia. Individuals who are high in social anhedonia have an innate tendency to withdraw from social interactions which potentially reduces the opportunity for them to build or gain subjectively meaningful social networks. Aside from a presumed reduced reward from social interactions, the reasons for social withdrawal in those with social anhedonia are poorly understood. We need to investigate whether individuals with high social anhedonia benefit from perceived social support across their lifespan despite their self-reported diminished pleasure from social interactions (Blanchard et al., 2011). In addition, we need to understand the preferred 
qualities of social support for those with social anhedonia in order to devise appropriate interventions to help alleviate their sense of disconnection. Those who score highly on social anhedonia do in fact get involved in romantic relationships despite facing struggles with their partners (Assad, \& Lemay, 2018). This suggests that those with social anhedonia do have some social and intimate needs. Absence of loneliness has been suggested to be the social equivalent of satisfying a basic physical need (Hawkley, \& Cacioppo, 2010), hence reinforcing the imperative drive for social connectedness (Cacioppo et al., 2006). Subsequently, this raises interesting questions concerning the qualities of the relationships which provide satisfaction for those with high levels of social anhedonia (Hawkley, \& Cacioppo, 2010).

From a broader perspective, exploring the underlying mechanisms influencing impaired social functioning has both research and clinical implications. First, recent research has emphasised that recovery from mental health disorders requires not only a significant decrease of clinical symptoms but also an improvement in domains including social skills and social roles (Brissos et al., 2011; Juckel, \& Morosini, 2008). Indeed, recovery in both domains may be necessary for sustained long term positive outcomes. In this context, social functioning is increasingly recognised as a treatment outcome for psychotherapy interventions, beyond symptomatic remission (Tan, Lee, \& Lee, 2018; Renner, Cuijpers, \& Huibers, 2014). Not only are improvements in social functioning found to correlate positively with engagement in interventions (Alvarez-Jimenez et al., 2018), but also with enhanced quality of life (Bonnin et al., 2019). In addition, functional recovery allows individuals to return to societal roles, which in turn, has been suggested to have the greatest impact on challenging stigma of mental illness (Corrigan, Morris, Michaels, Rafacz, \& Rusch, 2012; London, \& Evans-Lacko, 2010). Ultimately, it is increasingly important to consider social 
functioning in efforts to reduce the global burden of mental health disorders, particularly conditions that are chronic and debilitating (Rehm, \& Shield, 2019).

Some limitations in the current study should be addressed. First, due to the crosssectional design of the study, the temporal nature of the proposed relationships cannot be determined. We are viewing social anhedonia as relatively stable and trait-like on the basis of how it is treated in existing research (Loas, Monestes, Ingelaere, Noisette, \& Herbener, 2009; Olsen, Bjorkquist, Bodapati, Shankman, \& Herbener, 2015; Ritsner, 2016). Loneliness can fluctuate within an individual over the lifespan, although it is recognised that an individual can have an inherit trait-like tendency towards feeling lonely (Gao et al., 2017). Social functioning appears to be relatively stable; it can be changed, but often requires sustained effort and targeted interventions (De Silva, Cooper, Li, Lund, \& Patel, 2013; Roberts, \& Velligan, 2012). Second, the data in this study is collected predominantly from undergraduates, comprising mainly psychology students. This could reduce the generalisability of findings to the community sample; however, we did obtain a relatively large sample. In addition, there is no reason to suspect that psychology undergraduates might score unusually high or low on the variables under consideration in this study. Thirdly, the use of multiple objective methods of evaluation including parents and peer reports may minimize the influence of subjectivity, particularly for variables such as social functioning where self-ratings could potentially be biased especially for individuals with high levels of social anhedonia. This is important for future research to consider. Fourth, given the sex differences found for social functioning, mediation analyses should be run separately for both sexes. However, the number of males and females were not proportionate in this study to conduct such analyses purposefully. The reported sex differences do replicate previous studies that females have higher social functioning than males (Rietschel et al., 2017; Usall et al., 2007; Vaskinn et al., 2011). 
Despite these caveats, this study offers additional insights into current understanding of social anhedonia, loneliness and their association with social functioning in healthy and relatively high functioning adults. It is important to understand how social anhedonia, a transdiagnostic factor, relates to social functioning. Given the role loneliness plays in mediating social anhedonia effects on social functioning, this suggests that the alleviation of loneliness in many disorders, where social anhedonia is present, may help to improve overall social functioning. It is important to investigate how loneliness might mediate the effects of social anhedonia on social functioning. It is anticipated that this will serve as an impetus for future work on how social anhedonia is related to functioning, with a focus on the mediation pathways and conditional effects.

Conflict of interest: There is no competing interest to disclose

\section{References}

Alvarez-Jimenez, M., Gleeson, J. F., Bendall, S., Penn, D. L., Yung, A. R., Ryan, R. M., ... Nelson, B. (2018). Enhancing social functioning in young people at Ultra High Risk 
(UHR) for psychosis: A pilot study of a novel strengths and mindfulness-based online social therapy. Schizophrenia Research, 202, 369-377.

doi:10.1016/j.schres.2018.07.022

Assaad, L., \& Lemay, E. P. Jr. (2018). Social anhedonia and romantic relationship processes. Journal of Personality, 86, 147-157. doi:10.1111/jopy.12300

Badcock, J. C., Barkus, E., Cohen, A. S., Bucks, R., \& Badcock, D. R. (2016). Loneliness and schizotypy are distinct constructs, separate from general psychopathology. Frontiers in Psychology, 7(1018), 1-8. doi:10.3389/fpsyg.2016.01018

Badcock, J. C., Shah, S., Mackinnon, A., Stain, H. J., Galletly, C., Jablensky, A., \& Morgan, V. A. (2015). Loneliness in psychotic disorders and its association with cognitive function and symptom profile. Schizophrenia Research, 169, 268-273. doi:10.1016/j.schres.2015.10.027

Baumeister, R. F., \& Leary, M. R. (1995). The need to belong: Desire for interpersonal attachments as a fundamental human motivation. Psychological Bulletin, 117, 497529. doi:10.1037/0033-2909.117.3.497

Bedwell, J. S., Gooding, D. C., Chan, C. C., \& Trachik, B. J. (2014). Anhedonia in the age of RDoC. Schizophrenia Research, 160, 226-227. doi:10.1016/j.schres.2014.10.028

Birchwood, M., Smith, J., Cochrane, R., Wetton, S., \& Copestake, S. (1990). The Social Functioning Scale. The development and validation of a new scale of social adjustment for use in family intervention programmes with schizophrenia patients. British Journal of Psychiatry, 157, 853-859. doi:10.1192/bjp.157.6.853

Blanchard, J. J., Collins, L. M., Aghevli, M., Leung, W. W., \& Cohen, A. S. (2011). Social anhedonia and schizotypy in a community sample: the Maryland longitudinal study of schizotypy. Schizophrenia Bulletin, 37, 587-602. doi:10.1093/schbul/sbp107 
Blanchard, J. J., Gangestad, S. W., Brown, S. A., \& Horan, W. P. (2000). Hedonic capacity and schizotypy revisited: A taxometric analysis of social anhedonia. Journal of Abnormal Psychology, 109, 87-95. doi:10.1037//0021-843X.109.1.87

Blanchard, J. J., Horan, W. P., \& Brown, S. A. (2001). Diagnostic differences in social anhedonia: a longitudinal study of schizophrenia and major depressive disorder. Journal of Abnormal Psychology, 110, 363-371. doi:10.1037/0021-843X.110.3.363

Bonnin, C. D. M., Reinares, M., Martinez-Aran, A., Jimenez, E., Sanchez-Moreno, J., Sole, B., ... Vieta, E. (2019). Improving functioning, quality of life, and well-being in patients with bipolar disorder. International Journal of Neuropsychopharmacology, 22, 467-477. doi:10.1093/ijnp/pyz018

Brissos, S., Molodynski, A., Dias, V. V., \& Figueira, M. L. (2011). The importance of measuring psychosocial functioning in schizophrenia. Annals of General Psychiatry, 10(18), 1-7. doi:10.1186/1744-859X-10-18

Brown, L. H., Silvia, P. J., Myin-Germeys, I., \& Kwapil, T. (2007). When the need to belong goes wrong: The expression of social anhedonia and social anxiety in daily life. Psychological Science, 18, 778-782. doi:10.1111/j.1467-9280.2007.01978.x

Brown, L. H., Silvia, P. J., Myin-Germeys, I., Lewandowski, K. E., \& Kwapil, T. R. (2008). The relationship of social anxiety and social anhedonia to psychometrically identified schizotypy. Journal of Social and Clinical Psychology, 27, 127-149. doi:10.1521/jscp.2008.27.2.127

Burns, T., \& Patrick, D. (2007). Social functioning as an outcome measure in schizophrenia studies. Acta Psychiatrica Scandinavica, 116, 403-418. doi:10.1111/j.16000447.2007.01108.x 
Cacioppo, J. T., Hawkley, L. C., Ernst, J. M., Burleson, M., Berntson, G. G., Nouriani, B., \& Spiegel, D. (2006). Loneliness within a nomological net: An evolutionary perspective. Journal of Research in Personality, 40, 1054-1085. doi:10.1016/j.jrp.2005.11.007

Cicero, S. C., Krieg, A., Becker, T. M., \& Kerns, J. G. (2016). Evidence for the discriminant validity of the revised social anhedonia scale from social anxiety. Assessment, 23, 544-556. doi:10.1177/1073191115590851

Cornwell, E. Y., \& Waite, L. J. (2009). Social disconnectedness, perceived isolation, and health among older adults. Journal of Health and Social Behaviour, 50, 31-48. doi:10.1177/002214650905000103

Corrigan, P. W., Morris, S. B., Michaels, P. J., Rafacz, J. D., \& Rusch, N. (2012) Challenging the public stigma of mental illness: a meta-analysis of outcome studies. Psychiatric Services, 63, 963-973. doi:10.1176/appi.ps.201100529.

Danese, A., Moffitt, T. E., Harrington, H., Milne, B. J., Polanczyk, G., Pariante, C. M., . . Caspi, A. (2009). Adverse childhood experiences and adult risk factors for age-related disease: Depression, inflammation, and clustering of metabolic risk markers. Archives of Paediatrics \& Adolescent Medicine, 163, 1135-1143. doi:10.1177/1088868310377394

De Silva, M. J., Cooper, S., Li, H. L., Lund, C., \& Patel, V. (2013). Effect of psychosocial interventions on social functioning in depression and schizophrenia: meta- analysis. British Journal of Psychiatry, 202, 253-260. doi:10.1192/bjp.bp.112.118018

Dodell-Feder, D., Tully, L. M., Lincoln, S. H., \& Hooker, C. I. (2014). The neutral basis of theory of mind and its relationship to social functioning and social anhedonia in individuals with schizophrenia. NeuroImage: Clinical, 4, 154-163. doi:10.1016/j.nicl.2013.11.006 
Eckblad, M. L., Chapman, L. J., Chapman, J. P., \& Mishlove, M. (1982). The Revised Social Anhedonia Scale. Unpublished Test. University of Wisconsin, Madison.

Fortunati, R., Ossola, P., Camerlengo, A., Bettini, E., De Panfilis, C., Tonna, M., ... Marchesi, C. (2015). Anhedonia in schizophrenia: The role of subjective experiences. Comprehensive psychiatry, 62, 152-160. doi:10.1016/j.comppsych.2015.07.011

Frewen, P. A., Dozois, J. A., \& Lanius, R. A. (2012). Assessment of anhedonia in psychological trauma: psychometric and neuroimaging perspectives. European Journal of Psychotraumatology, 3, 8587-8598. doi:10.3402/ejpt.v3i0.8587

Gao, J., Davis, L. K., Hart, A. B., Sanchez-Roige, S., Han, L., Cacioppo, J. T., \& Palmer, A. A. (2017). Genome-wide association study of loneliness demonstrates a role for common variation. Neuropsychopharmacology, 42, 811-821. doi:10.1038/npp.2016.197

Gooding, D. C., Davidson, R. J., Putnam, K. M., \& Tallent, L. A. (2002). Normative emotion-modulated startle response in individuals at risk for schizophrenia-spectrum disorders. Schizophrenia Research, 57, 109-120. doi:10.1016/S0920-9964(01)00295$\mathrm{X}$

Gooding, D. C., Tallent, K. A., \& Matts, C. W. (2005). Clinical status of at-risk individuals 5 years later: further validation of the psychometric high-risk strategy. Journal of Abnormal Psychology, 114, 170-175. doi:10.1037/0021-843X.114.1.170

Harrison, A., Mountford, V. A., \& Tchanturia, K. (2014). Social anhedonia and work and social functioning in the acute and recovered phases of eating disorders. Psychiatry Research, 218, 187-194. doi:10.1016/j.psychres.2014.04.007

Hawkley, L. C., \& Cacioppo, J. T. (2010). Loneliness matters: A theoretical and empirical review of consequences and mechanisms. Annals of Behavioral Medicine, 40, $218-$ 227. doi:10.1007/s12160-010-9210-8 
Healey, K. L., Morgan, J., Musselman, S. C., Olino, T. M., \& Forbes, E. E. (2014). Social anhedonia and medial prefrontal response to mutual liking in late adolescents. Brain and Cognition, 89, 39-50. doi:10.1016/j.bandc.2013.12.004

Heinrich, L. M., \& Gullone, E. (2006). The clinical significance of loneliness: A literature review. Clinical Psychology Review, 26, 695-718. doi:10.1016/j.cpr.2006.04.002

Hellvin, T., Sundet, K., Vaskinn, A., Simonsen, C., Ueland, T., Andreassen, O. A., \& Melle, I. (2010). Validation of the Norwegian version of the social functioning scale (SFS) for schizophrenia and bipolar disorder. Scandinavian Journal of Psychology, 15, 525533. doi:10.1111/j.1467-9450.2010.00839.x

Henry, J. D., Bailey, P. E., \& Rendell, P. G. (2008). Empathy, social functioning and schizotypy. Psychiatry Research, 160, 15-22. doi:10.1016/j.psychres.2007.04.014

Holt-Lunstad, J., Smith, T. B., \& Layton, J. B. (2010). Social relationships and mortality risk: A meta-analytic review. PLoS Medicine, 7, 1-20. doi:10.1371/ journal.pmed.1000316

Horan, W. P., Brown, S. A., \& Blanchard, J. J. (2007). Social anhedonia and schizotypy: The contribution of individual differences in affective traits, stress, and coping. Psychiatry Research, 149, 147-156. doi:10.1016/j.psychres.2006.06.002

Horan, W. P., Green, M. F., Kring, A. M., \& Nuechterlein, K. H. (2006). Does anhedonia in schizophrenia reflect faulty memory for subjectively experienced emotions? Journal of Abnormal Psychology, 115, 496-508. doi:10.1037/0021-843X.115.3.496

House, J. S. (2001). Social isolation kills, but how and why? Psychosomatic medicine, 63, 273-274. doi:10.1097/00006842-200103000-00011

Jaremka, L. M., Andridge, R. R., Fagundes, C. P., Alfano, C. M., Povoski, S. P., Lipari, A. M., ... Kiecolt-Glaser, J. K. (2014). Pain, depression and fatigue: Loneliness as a longitudinal risk factor. Health Psychology, 33, 948-957. doi:10.1037/a0034012 
Joiner, T., Brown, J., \& Metalsky, G. (2003) A test of the tripartite model's prediction of anhedonia's specificity to depression: patients with major depression versus patients with schizophrenia. Psychiatry Research, 119, 243-250. doi:10.1016/S01651781(03)00131-8

Juckel, G., \& Morosini, P. L. (2008). The new approach: Psychosocial functioning as a necessary outcome criterion for the therapeutic success in schizophrenia. Current Opinion in Psychiatry, 21, 630-639. doi:10.1097/YCO.0b013e328314e144

Kwapil, T. R. (1998). Social anhedonia as a predictor of the development of schizophreniaspectrum disorders. Journal of Abnormal Psychology, 107, 558-565. doi:10.1037/0021-843X.107.4.558

Lavigne, G. L., Vallerand, R. J., \& Crevier-Braud, L. (2011). The fundamental need to belong: On the distinction between growth and deficit-reduction orientations. Personality and Social Psychology Bulletin, 37, 1185-1201. doi:10.1177/0146167211405995

Leifker, F. R., Patterson, T. L., Heaton, R. K., \& Harvey, P. D. (2011). Validating measures of real-world outcome: the results of the VALERO expert survey and RAND panel. Schizophrenia Bulletin, 37, 334-343. doi:10.1093/schbul/sbp044

Lim, M. H., \& Gleeson, J. F. (2014). Social connectedness across the psychosis spectrum: Current issues and future directions for interventions in loneliness. Frontiers in Psychiatry, 5, 1-3. doi:10.3389/fpsyt.2014.00154

Lim, M. H., Rodebaugh, T. L., Zyphur, M. J., \& Gleeson, J. F. M. (2016). Loneliness over time: The crucial role of social anxiety. Journal of Abnormal Psychology, 125, 620630. doi:10.1037/abn0000162 
Llerena, K., Park, S. G., Couture, S. M., \& Blanchard, J. J. (2012). Social anhedonia and affiliation: Examining behaviour and subjective reactions within a social interaction. Psychiatry Research, 200, 679-686. doi:10.1016/j.psychres.2012.07.050

Loas, G., Monestes, J. L., Ingelaere, A., Noisette, C., \& Herbener, E. S. (2009). Stability and relationships between trait or state anhedonia and schizophrenic symptoms in schizophrenia: A 13-year follow-up study. Psychiatry Research, 166, 132-140. doi:10.1016/j.psychres.2008.02.010

London, J., \& Evans-Lacko, S. E. (2010). Challenging mental health-related stigma through social contact. European Journal of Public Health, 20, 130-131. doi:10.1093/eurpub/ckq014

Lovibond, P. F., \& Lovibond, S. H. (1995a). The structure of negative emotional states: Comparison of the Depression Anxiety Stress Scales (DASS) with the Beck Depression and Anxiety Inventories. Behaviour Research and Therapy, 33, 335-343. doi:10.1016/0005-7967(94)00075-U

Lovibond, S. H., \& Lovibond, P. F. (1995b). Manual for the Depression Anxiety Stress Scales. Sydney: Psychology Foundation.

Martin, E. A., Cicero, D. C., Bailey, D. H., Karcher, N. R, \& Kerns, J. G. (2015). Social anhedonia is not just extreme introversion: Empirical evidence of distinct constructs. Journal of Personality Disorders, 29, 1-18. doi:10.1521/pedi_2015_29_203

Miller, G., Chen, E., \& Cole, S. W. (2009). Health psychology: Developing biologically plausible models linking the social world and physical health. Annual Review of Psychology, 60, 501-524. doi:10.1146/annurev.psych.60.110707.163551

Novacek, D. M., Gooding, D. C., \& Pflum, M. J. (2016). Hedonic Capacity in the Broader Autism Phenotype: Should Social Anhedonia Be Considered a Characteristic Feature? Frontiers in Psychology, 7, 1-8. doi:10.3389/fpsyg.2016.00666 
Olsen, E. K., Bjorkquist, O. A., Bodapati, A. S., Shankman, S. A., \& Herbener, E. S. (2015). Associations between trait anhedonia and emotional memory deficits in females with schizophrenia versus major depression. Psychiatry Research, 230, 323-330. doi:10.1016/j.psychres.2015.09.012

Peplau, L. A., \& Perlman, D. (1982). Perspectives on loneliness. In L. A. Peplau \& D. Perlman (Eds.), Loneliness: A sourcebook of current theory, research and therapy. New York, NY: Wiley-Interscience.

Preacher, K. J., \& Hayes, A. F. (2004). SPSS and SAS procedures for estimating indirect effects in simple mediation models. Behavior Research Methods, Instruments, \& Computers, 36, 717-731. doi:10.3758/BF03206553

Preacher, K. J., Rucker, D. D., \& Hayes, A. F. (2007). Addressing moderated mediation hypotheses: Theory, methods, and prescriptions. Multivariate Behavioral Research, 42, 185-227. doi:10.1080/00273170701341316

Rehm, J., \& Shield, K. D. (2019). Global burden of disease and the impact of mental and additive disorders. Current Psychiatry Reports, 21(10), 1-7. doi:10.1007/s11920-0190997-0

Renner, F., Cuijpers, P., \& Huibers, M. J. H. (2014). The effect of psychotherapy for depression on improvements in social functioning: a meta-analysis. Psychological medicine, 44, 2913-2926. doi:10.1017/S0033291713003152

Rey, G., Jouvent, R., \& Dubal, S. (2009). Schizotypy, depression, and anxiety in physical and social anhedonia. Journal of Clinical Psychology, 65, 695-708. doi:10.1002/jclp.20577

Rietschel, L., Lambert, M., Karow, A., Zink, M., Muller, H., Heinz, A., ... Bechdolf, A. (2017). Clinical high risk for psychosis: gender differences in symptoms and social functioning. Early Intervention in Psychiatry, 11, 306-313. doi:10.1111/eip.12240 
Ritsner, M. S. (2016). Anhedonia of patients with schizophrenia and schizoaffective disorder is attributed to personality-related factors rather than to state-dependent clinical symptoms. Clinical schizophrenia \& Related Psychoses, 9, 187-197. doi:10.3371/CSRP.RI.031513

Roberts, D. L., \& Velligan, D. I. (2012). Can social functioning in schizophrenia be improved through targeted social cognitive intervention? Rehabilitation Research and Practice, 2012, 1-8. doi:10.1155/2012/742106

Ross, S. R., Lutz, C. J., \& Bailley, S. E. (2002). Positive and negative symptoms of schizotypy and the Five-Factor Model: A domain and facet level analysis. Journal of Personality Assessment, 79, 53-72. doi:10.1207/S15327752JPA7901_04

Russell, D., Peplau, L. A., \& Cutrona, C. E. (1980). The revised UCLA Loneliness Scale: concurrent and discriminant validity evidence. Journal of Personality Assessment, 39, 472-480. Retrieved from https://www.ncbi.nlm.nih.gov/pubmed/7431205

Schürhoff, F., Szoke, A., Bellivier, F., Turcas, C., Villemur, M., Tignol, J., ... Leboyer, M. (2003). Anhedonia in schizophrenia: a distinct familial subtype? Schizophrenia research, 61, 59-66. doi:10.1016/S0920-9964(02)00237-2

Setterfield, M., Walsh, M., Frey, A. L., \& McCabe, C. (2016). Increased social anhedonia and reduced helping behaviour in young people with high depressive symptomatology. Journal of affective disorders, 205, 372-377. doi:10.1016/j.jad.2016.08.020

Spijker, J., Bijl, R. V., De Graaf, R., \& Nolen, W. A. (2001). Determinants of poor 1-year outcome of DSM-III-R major depression in the general population: Results of the Netherlands mental health survey and incidence study (NEMESIS). Acta Psychiatrica Scandinavica, 103, 122-130. doi:10.1034/j.1600-0447.2001.103002122.x 
Strauss, G. P., \& Gold, J. M. (2012). A new perspective on anhedonia in schizophrenia. The American Journal of Psychiatry, 169, 364-373. doi:10.1176/appi.ajp.2011.11030447

Tan, B. L., Lee, S. A., \& Lee, J. (2018). Social cognitive interventions for people with schizophrenia: A systematic review. Asian Journal of Psychiatry, 35, 115-131. doi:10.1016/j.ajp.2016.06.013

Timpano, K. R., Çek, D., Rubenstein, L. M., Murphy, D., \& Schmidt, N. B. (2014). Exploring the association between obsessive-compulsive symptoms and loneliness: Consideration of specificity and gender. Journal of Cognitive Psychotherapy: An International Quaterly, 28, 264-273. doi:10.1891/0889-8391.28.4.264

Treadway, M. T., \& Zald, D. H. (2011). Reconsidering anhedonia in depression: Lessons from translational neuroscience. Neuroscience and Biobehavioural Reviews, 35, 537555. doi:10.1016/j.neubiorev.2010.06.006

Uchino, B. N., Bowen, K., Carlisle, M., \& Birmingham, W. (2012). Psychological pathways linking social support to health outcomes: A visit with the 'ghosts' of research past, present and future. Social Science \& Medicine, 74, 949-957. doi:10.1016/j.socscimed.2011.11.023

Usall, J., Haro, M. J., Araya, S., Moreno, B., Munoz, P. E., Martinez, A., \& Salvador, L. (2007). Social functioning in schizophrenia: What is the influence of gender? European Journal of Psychiatry, 21, 199-205. doi:10.4321/S021361632007000300004

Vaskinn, A., Sundet, K., Simonsen, C., Hellvin, T., Melle, I., \& Andreassen, O. A. (2011). Sex differences in neuropsychological performance and social functioning in schizophrenia and bipolar disorder. Neuropsychology, 25, 499-510. doi: $10.1037 / \mathrm{a} 0022677$ 
Velthorst, E., Nieman, D. H., Becker, H. E., van de Fliert, R., Dingermans, P. M., Klaasssen, R., ... Linszen, D. H. (2009). Baseline differences in clinical symptomology between ultra-high risk subjects with and without a transition to psychosis. Schizophrenia Research, 109, 60-65. doi:10.1016/j.schres.2009.02.002 
Table 1

Participants demographics and Means [Standard Deviations] of Clinical Variables $(n=824)$

$\begin{array}{lc}\text { Age (years) } & 21.03(5.59) \\ \% \text { Male } & 27.7 \%\end{array}$

Social Anhedonia (RSAS)

$16.49(7.24)$

Loneliness (UCLA)

$42.99(10.29)$

Social Functioning (SFS)

$149.55(22.17)$

Negative Affect (DASS)

$15.92(11.99)$ 
Table 2

Pearson correlation matrix between Social Anhedonia, Loneliness, Social Functioning (overall and subscales) and Negative Emotional State $(N=824)$.

$\begin{array}{lllllllllll}\text { Variables } & 1 & 2 & 3 & 4 & 5 & 6 & 7 & 8 & 9 & 10\end{array}$

1. RSAS

2. UCLA

$.41^{*}$

3. SFS

$-.20 *-.43 *-$

4. Withdrawal

$$
-.33^{*}-.35 * \quad .42 * \quad-
$$

5. Interpersonal

$$
-.64 *-.46 * \quad .35 * \quad .49 * \quad-
$$

6. Social Activities

$$
\begin{array}{lllll}
-.38 * & -.41 * & .51 * & .31 * \quad .48 *
\end{array}
$$

7. Recreation

$$
\begin{array}{lllllll}
-.51 * & -.20 * & .38 * & .36 * & .64 * & .60 * & -
\end{array}
$$
Activities

$\begin{array}{llllllll}-.12 * & -.19 * & .26 * & .17 * & .13 * & .04 & .06 & -\end{array}$ Competence

9. Independence Performance

$$
\begin{array}{llllllll}
-.58 * & -.18 * & .33 * & .37 * & .67 * & .42 * & .70 * & .28 *
\end{array}
$$$$
\begin{array}{lllllllll}
.33 * & .50 * & -.35^{*} & -.33^{*} & -.29 * & -.24 * & -.14 * & -.21^{*} & -.17 *
\end{array}
$$ 
Table 3 Results of the mediation analyses investigating loneliness as a mediator between social anhedonia and subscales of social functioning

\begin{tabular}{|c|c|c|c|}
\hline SA-UCLA-SF & $B$ & $\mathrm{SE}$ & $t$ \\
\hline Effect of SA on UCLA ${ }^{a}$ & .38 & .04 & $8.86^{* * *}$ \\
\hline Effect of UCLA on SF ${ }^{a}$ & -.73 & .08 & $-9.05^{* * *}$ \\
\hline Total effect of SA on $\mathrm{SF}^{\mathrm{a}}$ & -.29 & .11 & $-2.74 * *$ \\
\hline \multirow[t]{2}{*}{ Direct effect of SA on SF } & -.01 & .11 & -.07 \\
\hline & Bootstrap estimate & $\mathrm{SE}$ & BC $95 \%$ CI $[\mathrm{LL} ; \mathrm{UL}]^{\mathrm{b}}$ \\
\hline Indirect effects of SA on SF through UCLA & -.28 & .04 & {$[-.37 ;-.21]$} \\
\hline SA-UCLA-Withdrawal & $B$ & SE & $t$ \\
\hline Effect of SA on UCLA ${ }^{a}$ & .38 & .04 & $8.86^{* * *}$ \\
\hline Effect of UCLA on Withdrawal ${ }^{\mathrm{a}}$ & -.04 & .01 & $-4.81 * * *$ \\
\hline
\end{tabular}


Total effect of SA on Withdrawal ${ }^{\mathrm{a}}$

Direct effect of SA on Withdrawal

Indirect effects of SA on Withdrawal through UCLA

SA-UCLA-Interpersonal

Effect of SA on UCLA ${ }^{\mathrm{a}}$

Effect of UCLA on Interpersonal

Total effect of SA on Interpersonal ${ }^{\mathrm{a}}$

Direct effect of SA on Interpersonal

Indirect effects of SA on Interpersonal through UCLA

\begin{tabular}{ccc}
\hline Bootstrap estimate & SE & BC 95\% CI [LL;UL] \\
\hline-.02 & .004 & {$[-.03 ;-.01]$} \\
\hline$B$ & SE & $t$ \\
\hline .38 & .04 & $8.86^{* * *}$ \\
-.12 & .02 & $-7.81^{* * *}$ \\
-.43 & .02 & $-21.68 * * *$ \\
-.39 & .02 & $-19.16^{* * *}$ \\
\hline Bootstrap estimate & SE & BC 95\% CI [LL;UL] ${ }^{\mathrm{b}}$ \\
\hline-.05 & .01 & {$[-.06 ;-.03]$} \\
\hline
\end{tabular}

.01

$-7.20 * * *$

$-.07$

.01

$-5.55^{* * *}$ 
SA-UCLA-Social Activities

Effect of SA on UCLA

Effect of UCLA on Social Activities

Total effect of SA on Social Activities ${ }^{\mathrm{a}}$

Direct effect of SA on Social Activities

Indirect effects of SA on Social Activities through UCLA

SA-UCLA-Recreational Activities

Effect of SA on UCLA ${ }^{\mathrm{a}}$

Effect of UCLA on Recreational activities

Total effect of SA on Recreational activities
SE

.04

.04

.06

.06

\begin{tabular}{ccc}
\hline Bootstrap estimate & SE & BC 95\% CI [LL;UL] \\
\hline-.14 & .02 & {$[-.19 ;-.10]$} \\
$B$ & SE & $t$
\end{tabular}

.38

.04

$8.86^{* * *}$

.04

.01

$-.72$

$8.86 * * *$

-8.02 ***

$-10.13^{* * *}$

$-7.67 * * *$ 
Direct effect of SA on Recreational activities

Indirect effects of SA on Recreational activities through UCLA

\section{SA-UCLA- Independence Competence}

Effect of SA on UCLA

Effect of UCLA on Independence Competence ${ }^{a}$

Total effect of SA on Independence Competence ${ }^{\mathrm{a}}$

Direct effect of SA on Independence Competence through UCLA

\begin{tabular}{|c|c|c|}
\hline-.72 & .05 & $-15.74 * * *$ \\
\hline Bootstrap estimate & SE & BC $95 \%$ CI $[\mathrm{LL} ; \mathrm{UL}]^{\mathrm{b}}$ \\
\hline .00 & .01 & {$[-.03 ; .03]$} \\
\hline$b$ & $\mathrm{SE}$ & $t$ \\
\hline .38 & .04 & $8.86 * * *$ \\
\hline-.04 & .02 & -2.5 \\
\hline-.03 & .02 & -1.65 \\
\hline-.02 & .02 & -.84 \\
\hline Bootstrap estimate & $\mathrm{SE}$ & BC 95\% CI [LL;UL] $]^{\mathrm{b}}$ \\
\hline-.01 & .01 & {$[-.03 ;-.00]$} \\
\hline
\end{tabular}




\section{SA-UCLA- Independence Performance}

Effect of SA on UCLA ${ }^{\mathrm{a}}$

Effect of UCLA on Independence Performance

Total effect of SA on Independence Performance

Direct effect of SA on Independence Performance

Indirect effects of SA on IndPerf through UCLA $b$

.38

.06

$-.69$

$-.71$

.04

SE

BC $95 \%$ CI $[\mathrm{LL} ; \mathrm{UL}]^{\mathrm{b}}$
SE

.04

.03

.04
.01

$[.00 ; .05]$

Note. aDASS is a significant covariate for the pathway, $p<.01$ bower and upper limit of bias-corrected 95\% confidence interval with 10,000 bootstrap samples; $\mathrm{SA}=$ Social anhedonia; UCLA =Loneliness; $\mathrm{SF}=$ Social Functioning. ${ }^{*} p<.05 ; * * p<.01 ; * * * p<.001$. Significant mediation statistics are in bold. 
Figure captions

Figure 1. A schematic model with loneliness as the mediator in the relationship between social anhedonia (SA) and social functioning (SF). Path $c$ represents the total effect of SA on SF (without considering mediating effect of loneliness). Path $a$ refers to the direct effect of SA on

loneliness. Path $b$ represents the direct impact of loneliness on SF after controlling for SA. Path $c$ ' depicts the direct impact of SA on SF after controlling for loneliness, the mediator. The indirect effect of SA on SF via loneliness is calculated as the product of the direct effects of SA on loneliness and loneliness on SF ( $a \mathrm{X} b)$. 

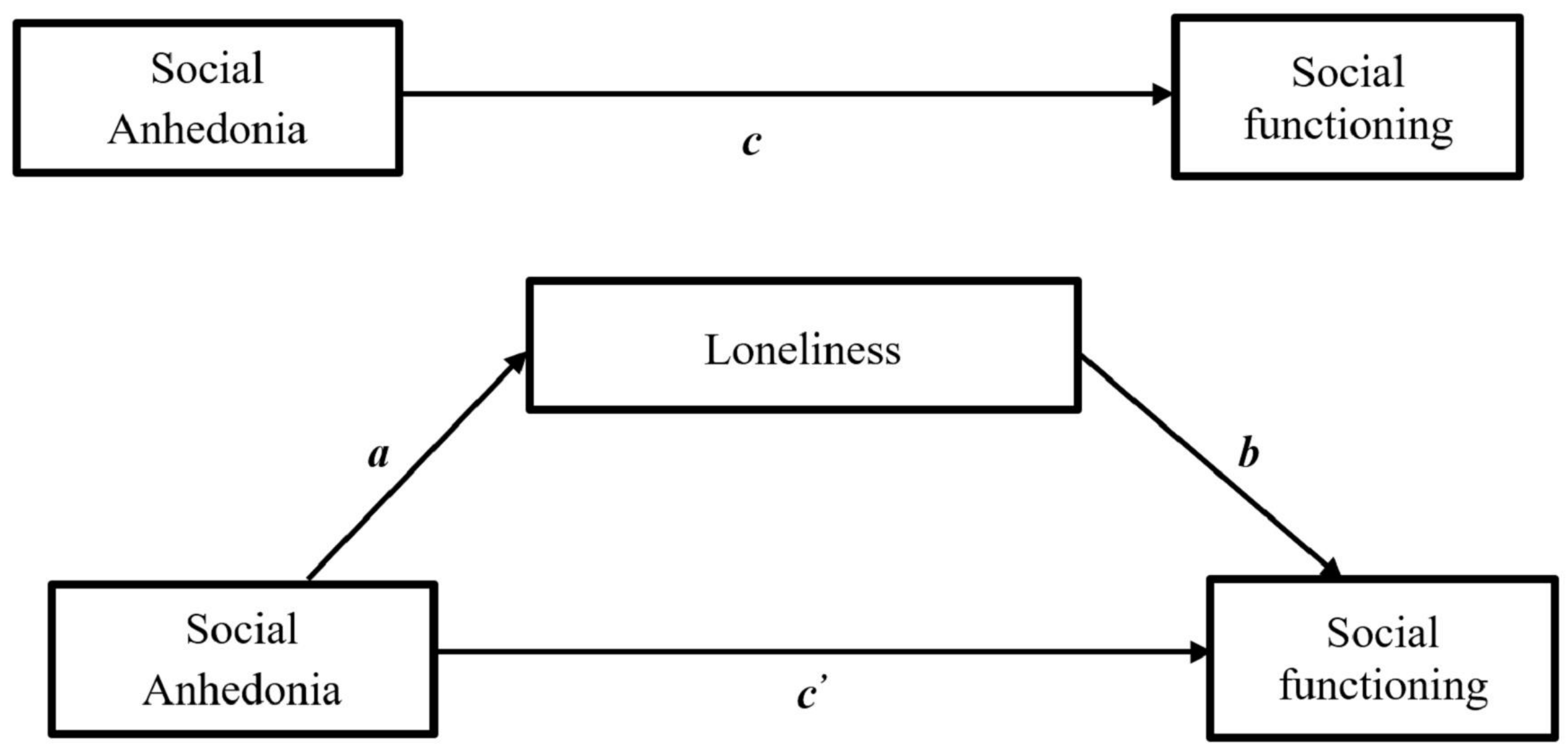